\title{
Energy Management System for Hybrid PV-Wind-Battery Microgrid using Convex Programming, Model Predictive and Rolling Horizon Predictive Control with Experimental Validation
}

\author{
Mahmoud Elkazaz ${ }^{a, b}$, Mark Sumner ${ }^{a}$, David Thomas ${ }^{a}$ \\ ${ }^{a}$ Department of Electrical \& Electronic Engineering, The University of Nottingham, Nottingham NG72RD, UK \\ ${ }^{b}$ Department of Electrical Power \& Machines Engineering, Tanta University, Tanta 31511, Egypt.
}

\author{
ARTICLE INFO \\ Article history: \\ Received 00 xxxxx 00 \\ Received in revised form $00 \mathrm{xxxxx} 00$ \\ Accepted 00 xxxxxx 00 \\ Keywords: \\ Microgrid Energy management \\ Battery energy storage system \\ Real-time battery control \\ Convex optimization \\ Model predictive control \\ Rolling Horizon Predictive \\ Controller \\ Adaptive Autoregression \\ Algorithm
}

\begin{abstract}
A B S T R A C T
The integration of energy storage technologies with renewable energy systems can significantly reduce the operating costs for microgrids (MG) in future electricity networks. This paper presents a novel energy management system (EMS) which can minimize the daily operating cost of a MG and maximize the self-consumption of the RES by determining the best setting for a central battery energy storage system (BESS) based on a defined cost function. This EMS has a two-layer structure. In the upper layer, a Convex Optimization Technique is used to solve the optimization problem and to determine the reference values for the power that should be drawn by the MG from the main grid using a 15 minute sample time. The reference values are then fed to a lower control layer, which uses a 1 minute sample time, to determine the settings for the BESS which then ensures that the MG accurately follows these references. This lower control layer uses a Rolling Horizon Predictive Controller and Model Predictive Controllers to achieve its target. Experimental studies using a laboratory-based MG are implemented to demonstrate the capability of the proposed EMS.
\end{abstract}

(C) 20xx xxxxxxxx. Hosting by Elsevier B.V. All rights reserved.

\section{Introduction}

The growth of renewable energy sources (RES) in the electrical grid together with the increasing use of electricity for transport and heating, ventilation and air-conditioning require a new vision for future transmission and distribution grids. Increasing the complexity and variability of generation introduces challenges for the electricity system, and innovative technologies are required to maintain stable and reliable system management [1].

Microgrids (MG) which combine different kinds of distributed energy resources (DERs) such as distributed generators, distributed storage units, as well as different types of load and control devices are a promising structure for future electric networks [2],[3]. MGs are capable of managing and coordinating their own DERs in a more decentralized way, which reduces the need for centralized coordination and management. From this point of view, the optimization of the MG operation to minimize operating costs and increase the efficiency of its energy resources has now become extremely important [4], [5].
Nomenclature

$C_{O_{-} M G} \quad$ Daily operating cost of the MG (£/day).

$C_{M G \_b u y} \quad$ Daily cost of the electrical energy purchased from the main electrical grid (£/day).

$C_{M G_{-} \text {sell }} \quad$ Daily income from the exported electrical energy to the main electrical grid (£/day).

$C_{\text {BESS_op }}$ Daily operation and maintenance cost of the BESS.

$P_{\text {Utility }}(t) \quad$ Daily electric power drawn by the MG from the main electric grid $(\mathrm{kW})$.

$\Delta T \quad$ UEML sampling time (h) (i.e. $15 / 60$ hour).

Tariff $_{\text {buy }}(t)$ Electricity purchase tariff for the energy drawn from the main grid $(£ / \mathrm{kWh})$.

Tariff $_{\text {sell }}(t)$ Tariff for selling the excess electric energy to the main electrical grid $(£ / \mathrm{kWh})$.

$P_{B E S S}(t) \quad$ The electrical power charged/discharged by the BESS at a time interval " $\mathrm{t}$ " $(\mathrm{kW})$.

$P_{b}(t) \quad$ The discharge/charge power from/to the battery at a time interval $\mathrm{t}(\mathrm{kW})$ : where a +ve value denotes battery discharging, and a -ve value denotes charging.

$E(t) \quad$ Stored energy in the BESS at a time interval $\mathrm{t}$ $(\mathrm{kWh})$. 
$E(t-1) \quad$ Stored energy in the BESS at a time interval t-1 (kWh).

$\eta_{d}, \eta_{c} \quad$ Efficiencies of the battery discharging and charging respectively (\%).

$B_{\text {Capacity }} \quad$ Battery capacity $(\mathrm{kWh})$.

$\eta_{\text {Conv }} \quad$ Power converter efficiency (\%).

$P_{\text {con_const }}$ Constant power losses in the power converter $(\mathrm{kW})$.

$\operatorname{SOC}(t) \quad$ State of charge of the battery at a time interval t (\%).

$P_{\text {Utility }}(t) \quad$ Electric power drawn by the MG from the main electrical grid at a time interval $\mathrm{t}(\mathrm{kW})$ : where a +ve means the MG imports power from the main grid, and a -ve means the MG exports power to the main grid.

$P_{\text {load }}(t) \quad$ Electrical load demand of the MG at a time interval $\mathrm{t}(\mathrm{kW})$.

$P_{P V}(t) \quad$ Electric power generated by the PV system located at the $\mathrm{MG}$ at a time interval $\mathrm{t}(\mathrm{kW})$.

$P_{\text {wind }}(t) \quad$ Electric power generated by the wind turbine located at the $\mathrm{MG}$ at a time interval $\mathrm{t}(\mathrm{kW})$.

$P_{B E S S \max } \quad$ Maximum power that can be produced by the BESS at time interval $\mathrm{t}(\mathrm{kW}),+\mathrm{P}$ means the maximum discharge power, $-\mathrm{P}$ means the maximum charge power.

$S O C_{\max } \quad$ Maximum state of charge limits of the BESS

$S O C_{\min } \quad$ Minimum state of charge limits of the BESS

$\triangle P_{B E S S}(t) \quad$ Variation of the BESS power output between two consecutive time slots $(\mathrm{kW})$.

$\triangle P_{B E S S_{\max }} \quad$ Maximum acceptable change of the BESS power output for both charging and discharging stages $(\mathrm{kW})$.

Pnet $_{\text {Forecaste, }}$ Forecasted net demand of the MG (kW); (i.e. obtained from AR forecasting and used in LCL)

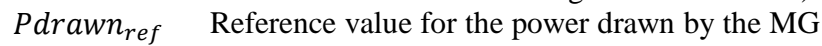
from the main electric grid in the next sample time $(\mathrm{kW})$ (i.e. obtained from the UEML).

$C C_{\text {bat }} \quad$ Capital cost of the BESS

$N_{\text {cycle }} \quad$ Number of life cycles of the BESS

$C_{\text {deg }} \quad$ Factor to penalize the degradation process of the batteries due to high stress in the charging and discharging process.

$P_{b, \text { total }}(t) \quad$ Net power produced by the BESS (P discharge $P$ charge)

Abbreviations

EMS Energy management system

MG Microgrid

RES Renewable energy resources

DERs Distributed energy resources

MGEM Microgrids energy management

UEML Upper energy management layer

LCL Lower control layer

MILP Mixed integer linear programming

BESS Battery energy storage system

ESS Energy storage system

TOU Time of use tariff

PV Photovoltaic

SOC State of charge

CREST Center for renewable energy systems

RHPC Rolling horizon predictive controller

MPC Model predictive controller

VSTEF Very short term energy forecasting

ANFIS Adaptive neuro fuzzy inference system

AR Autoregression forecasting algorithm

CAN Controller Area Network
To achieve interactive operation of RES and the other MG components, an Energy Management System (EMS) is required [6], [7]. The EMS controls the power flow within the MG by providing reference profiles for the controllers in the MG based on predefined objectives [8].

The current trend in microgrids is oriented towards encouraging local consumption of the energy generated by RES rather than exporting the surplus electric energy to the main grid [9]. This trend has received more attention following the development of new energy storage technologies and techniques for merging energy storage systems (ESS) into the MG architecture. Storage systems will play a key role in future electric grid operation [10]. Most grid operators are encouraging the use of ESS to address the increasing peak demand for electrical energy and congestion in the electricity grid [11], [12].

There is much research focusing on microgrid energy management (MGEM), particularly in optimizing system behavior. [13] introduces a power demand task scheduling policy that minimizes the MG operational costs over a fixed time horizon. The cost is a convex function of total instantaneous power consumption. Numerical results demonstrate the benefit of the proposed approaches compared to the default policy of serving demands. The authors in [14] focused on introducing a novel two-stage stochastic energy management to minimize the operational cost of a microgrid with various types of distributed energy resources. A scenario reduction method based on mixedinteger linear optimization was used to obtain the set of reduced scenarios. The authors took the uncertainty of price, load, wind speed and solar radiation into account in order to obtain more realistic results. The use of a scenario reduction method based on MILP optimization is often used offline, which restricts its use for real-time applications especially when dealing with demand-side management. In [15], the authors developed an algorithm that decomposes and solves the online problem in a distributed manner and proves that the distributed online solution is asymptotically optimal. The problem is shown to be convex and can be solved with a centralized online algorithm. The authors in [16] focused on the development of optimization-based scheduling strategies for the coordination of MGs. Simultaneous management of energy demand and energy production are used within a reactive scheduling approach to solve the problem of uncertainty associated with generation and consumption. In [17] and [18], the authors introduced a new distributed control scheme, which can achieve stable and optimal load sharing among multiple permanent magnet synchronous generators (PMSGs) in a DC microgrid based on distributed model predictive control (MPC). This scheme has a two-layer structure. The upper layer controllers coordinate the operation of parallel-connected grid-side converters and provide power references for each PMSG. The lower layer controls the PMSG.

The real-time EMS has received a lot of attention in the literature. A novel MGEM system based on a two-stage rolling horizon $(\mathrm{RH})$ strategy for a renewable-based $\mathrm{MG}$ is proposed in [19] and implemented for an MG, which consists of two wind turbines, photovoltaic panels, a diesel generator, and an energy storage system. However, the authors used 
economic load dispatch (ELD) with a sample time of 5 minutes in the rolling horizon stage. No results were presented to demonstrate the performance of the system using the ELD: the results presented have a 1 hour sample time. The authors in [20] applied a MPC approach to the problem of efficiently optimizing MG operation while satisfying a timevarying request and operating constraints. The results show a significant improvement in the quality of the solution and the computational burden. Martin et al. [21] presented an EMS prototype for an isolated renewable-based MG which consists of two stages: a deterministic management model was formulated in the first stage followed by its integration into a Rolling Horizon ( $\mathrm{RH}$ ) control strategy. The advantage of this proposal is that it considers the management of energy sources and can potentially include flexible timing of energy consumption. In [22], a day-ahead Economic Load Dispatch (ELD) was performed for a microgrid with intermittent DGs and an ESS; it was adjusted every 15 minutes to ensure that the voltages were kept within acceptable limits, trying to maintain the dispatch of units as close as possible to the predetermined values. The effect of using a shorter sample time on the economic results has not been studied compared to other cases in which a longer sample time is used. A more detailed formulation is presented in [23] for a microgrid with wind turbines and a hydrogen-based ESS, where the ELD is performed over several time steps, but only the results obtained for the next time step are actually implemented in the microgrid, and then the ELD is re-calculated for the following stages using a Model Predictive Control. In [24], an adaptable MGEM is designed and implemented experimentally for an online scheme. In this case, the author aims to minimize the operating costs and the disconnection of loads by proposing an architecture that allows the interaction of forecasting, measurement and optimization modules.

In [25] and [26], the benefits from optimal management of the ESS via multi-stage optimization are estimated to be a reduction of $5 \%$ in the operation cost, although this result strongly depends on the particular size and efficiency of the ESS considered, and the cost characteristics of the microgrid generators. A sample time of 1 hour has been used in this study. [27], [28] and [29] present an energy management strategy based on a low complexity Fuzzy Logic Control for grid power profile smoothing of a residential grid-connected microgrid including Renewable Energy Sources (RES) and Battery Energy Storage System (ESS). Although the proposed strategy shows good results, the effect of the proposed EMS on the battery lifetime and battery state of health have not been considered.

This paper introduces and implements an energy management system for microgrids. The MGEM system aims to: (1) Minimize the daily operating costs of the MG and enhance the local self-consumption of the renewable energy resources (RES) of the MG: (2) Smooth the power profile for the power drawn by the MG from the main grid: (3) Control the BESS with high accuracy through the proper design and implementation of a suitable controller: the Rolling Horizon Predictive controller (RHPC) and the model predictive controller (MPC), to compensate for any change in load and generation through the day. Experimental studies are presented using a laboratory-based MG to demonstrate the capability of the proposed EMS for real-time control.
The proposed MGEM system consists of a two-layer structure: the Upper Energy Management Layer (UEML), and the lower control layer (LCL).

(1) In the UEML a cost function is defined which minimizes the daily operating cost of the MG. The optimization problem (including constraints) has to be solved using an appropriate optimization technique which determines the appropriate reference values for the power drawn by the MG from the main grid, to minimize the daily MG operating cost. The optimization process uses a 15 minutes sample time and is repeated every 15 minutes in a rolling horizon manner.

(2) In the LCL two types of controllers - a Rolling Horizon Predictive Controller (RHPC) and a Model predictive controller (MPC) - are used to determine the settings for the BESS so that the MG accurately follows the reference values obtained from the UEML and smoothes the power profile for the power drawn by the MG from the main grid. These controllers use a one minute sample time to ensure very accurate results.

This paper contributes to the energy management of the MGs by introducing a very short sample time EMS. The structure of the two-layer EMS enables the algorithm to deal with frequent changes in the system using a very short sample time (i.e. 1 minute). This short sample time enables the proposed EMS to observe and respond to the small changes in load and generation throughout the day: this is a considerable challenge as a large amount of data must be processed and responded to in a short sample time. Much of the research published in the context of MGEM tends to use sampling times ranging from 15 minutes to 2 hours. The benefits of this EMS are that it reduces the dependency of the MG on the main electrical grid (by increasing selfconsumption of locally generated energy), reduces energy costs for end-users and the MG consumption profile can be shaped to reduce consumption peaks by appropriate selection of TOU tariff periods. Using RTPC/MPC as a part of the MGEM system has three benefits. It forces the MG to accurately follow the reference values for the power drawn from the main electric grid, it smoothes the power profile for the power drawn by the MG from the main grid, and the RTPC/MPC can help the MG to follow the reference values received from the main grid directly when being operating as a part of a community microgrids.

Also, a comparison between the performance of RHPC and MPC has been made to demonstrate the capability and the robustness of the RHPC to control the BESS with very high accuracy even when using a very short sample time. Furthermore, the RHPC does not require the complex optimization process that the MPC uses.

The paper is arranged as follows: section II gives a full description of the MG used. Section III focuses mainly on the Upper Energy Management Layer of the proposed EMS. This section introduces the formulation of the optimization problem including the cost function and the constraints. It also introduces the optimization technique used to solve the proposed optimization problem. Section IV focus on the lower control layer in which the RHPC and MPC are used to control of the BESS. In section V, experimental results for the proposed strategy are provided. 


\section{Microgrid architecture}

The MG used in this paper is assumed to be a UK based community fed from a Photovoltaic (PV) generation station, a wind turbine and a BESS. The MG is also assumed to be connected to the main electric grid to import the extra energy needed. The average load demand for the community used is assumed to be $1920 \mathrm{kWh}$ and the load profiles are created using a model from the Centre for Renewable Energy Systems Technology (CREST) created by Richardson and Thompson [30] based on actual measurements. The PV generation station used is the $22 \mathrm{~kW}$ station at the Energy Technology Building located at the University of Nottingham: additional PV generation profiles used are real profiles available at the PVOutput website [31]. The wind turbine used in this research is a $100 \mathrm{~kW}$ turbine located in Belgium, and the real daily wind power generation profiles are available at the Elia website [32]. The energy management hierarchy of the MG is shown in Fig. 1.

\section{Upper Energy Management Layer}

The function of this layer is to minimize the daily operating costs of the MG and enhance the self-consumption of the RES of the MG. This is achieved by the formulation of the optimization problem including the cost function and the constraints. The optimization problem (including constraints) is solved using a Convex optimization technique which determines the appropriate reference values for the power drawn by the MG from the main grid, to minimize the daily MG operating cost. This layer is updated every 15 minutes, in which more accurate forecasted load profiles, PV, Wind power generation are used, the updated BESS SOC value is measured, and new references values are obtained.

\subsection{Cost function formulation}

The cost function is formulated to minimize the daily operating cost of the $\mathrm{MG}$ " $\mathrm{C}_{O_{P} M G}$ " and to increase the selfconsumption of the RES located within the MG. This cost can be developed in terms of: (1) payments (i.e. the cost of purchased electricity from the main grid), (2) Incomes (i.e. considering the revenue of the excess energy sold to the main grid produced by the MG PV and wind generation after satisfying the MG's demand and charging the BESS) [33], (3) Daily operation costs of the BESS. The daily operating cost of the BESS is calculated based on the daily operation scenario of the BESS and also the effect of the BESS degradation [34],[35].

The daily operating cost of the MG is formulated as follows:

$$
\mathrm{C}_{O P \_M G}=C_{M G \_b u y}+C_{M G \_s e l l}+C_{B E S S_{-} \text {op }}
$$

$C_{M G \_b u y}$

$$
=\left\{\begin{array}{cl}
\Delta \mathrm{T} \times \sum_{t o}^{\mathrm{T}} \text { Tariff }_{\text {buy }}(\mathrm{t}) \times \mathrm{P}_{\text {Utility }}(t), & \mathrm{P}_{\text {Utility }}(t)>0 \\
0 & , \mathrm{P}_{\text {Utility }}(t) \leq 0
\end{array}\right.
$$

$$
\begin{aligned}
& C_{M G \_s e l l} \\
& =\left\{\begin{array}{cl}
\Delta \mathrm{T} \times \sum_{t o}^{\mathrm{T}} \text { Tariff }_{\text {sell }}(\mathrm{t}) \times \mathrm{P}_{\text {Utility }}(t) & , \mathrm{P}_{\text {Utility }}(t)<0 \\
0 & , \mathrm{P}_{\text {Utility }}(t) \geq 0
\end{array}\right. \\
& C_{B E S S_{o p}}=\sum_{t o}^{\mathrm{T}} \frac{\mathrm{CC}_{\mathrm{bat}} * \eta_{\mathrm{c}} * \Delta \mathrm{T} * \mathrm{P}_{\mathrm{b}, \text { charge }}(\mathrm{t})}{2 * \mathrm{~N}_{\text {cycle }}} \\
& +\frac{\mathrm{CC}_{\mathrm{bat}} * \Delta \mathrm{T} * \mathrm{P}_{\mathrm{b}, \text { disch }}(\mathrm{t})}{\eta_{\mathrm{d}} * 2 * \mathrm{~N}_{\text {cycle }}}+\mathrm{C}_{\mathrm{deg}} \\
& \text { * } \mathrm{P}_{\mathrm{b}, \text { total }}(\mathrm{t})
\end{aligned}
$$

\subsection{MG modelling and formulation of constraints}

The equations that represent the economic model of the MG, as well as all the constraints associated with its operation, are formulated as follows:

\subsubsection{MG active power balance equation}

The balance equation of the total active power in the MG is formulated as follows:

$$
\mathrm{P}_{\text {Utility }}(t)=\mathrm{P}_{\text {load }}(t)-\mathrm{P}_{\mathrm{PV}}(t)-\mathrm{P}_{\text {wind }}(t)-\mathrm{P}_{\mathrm{BESS}}(t)
$$

\subsubsection{Battery energy storage system model}

The BESS used in this research is represented by the following equations [36], [37]:

$$
\begin{gathered}
E(t)= \begin{cases}E(t-1)-\frac{\Delta \mathrm{T} \times \mathrm{P}_{\mathrm{b}}(\mathrm{t})}{\eta_{\mathrm{d}}} & , \mathrm{P}_{\mathrm{b}}(\mathrm{t})>0 \\
E(t-1)-\Delta \mathrm{T} \times \eta_{\mathrm{c}} \times \mathrm{P}_{\mathrm{b}}(t) & , \mathrm{P}_{\mathrm{b}}(\mathrm{t}) \leq 0\end{cases} \\
\operatorname{SOC}(\mathrm{t})=\frac{\mathrm{E}(\mathrm{t})}{\mathrm{B}_{\text {Capacity }}}
\end{gathered}
$$

A power converter is a part of the BESS. It is used to control the battery and acts as an interface between the BESS and the MG. The following equation represents the model of the power converter used in this research:

$$
\mathrm{P}_{\text {BESS }}(\mathrm{t})=\left\{\begin{array}{cl}
\mathrm{P}_{\mathrm{b}}(\mathrm{t}) \times \eta_{\text {Conv }}-\mathrm{P}_{\text {con_const }}, & \mathrm{P}_{\mathrm{b}}(\mathrm{t})>0 \\
\frac{\mathrm{P}_{\mathrm{b}}(\mathrm{t})}{\eta_{\text {Conv }}}+\mathrm{P}_{\text {con_const }} & , \mathrm{P}_{\mathrm{b}}(\mathrm{t}) \leq 0
\end{array}\right.
$$

The following equations represent the constraints used in this work. The constraints reflect the limits of the generation units within the MG and also define the operating range of the MG [33].

- Constraint of BESS power output

$$
-\mathrm{P}_{\text {BESS max }} \leq \mathrm{P}_{\text {BESS }}(\mathrm{t}) \leq \mathrm{P}_{\text {BESS max }}
$$

- Constraint of BESS State of charge (SOC)

$$
\mathrm{SOC}_{\min } \leq \mathrm{SOC}(\mathrm{t}) \leq S O C_{\text {max }}
$$




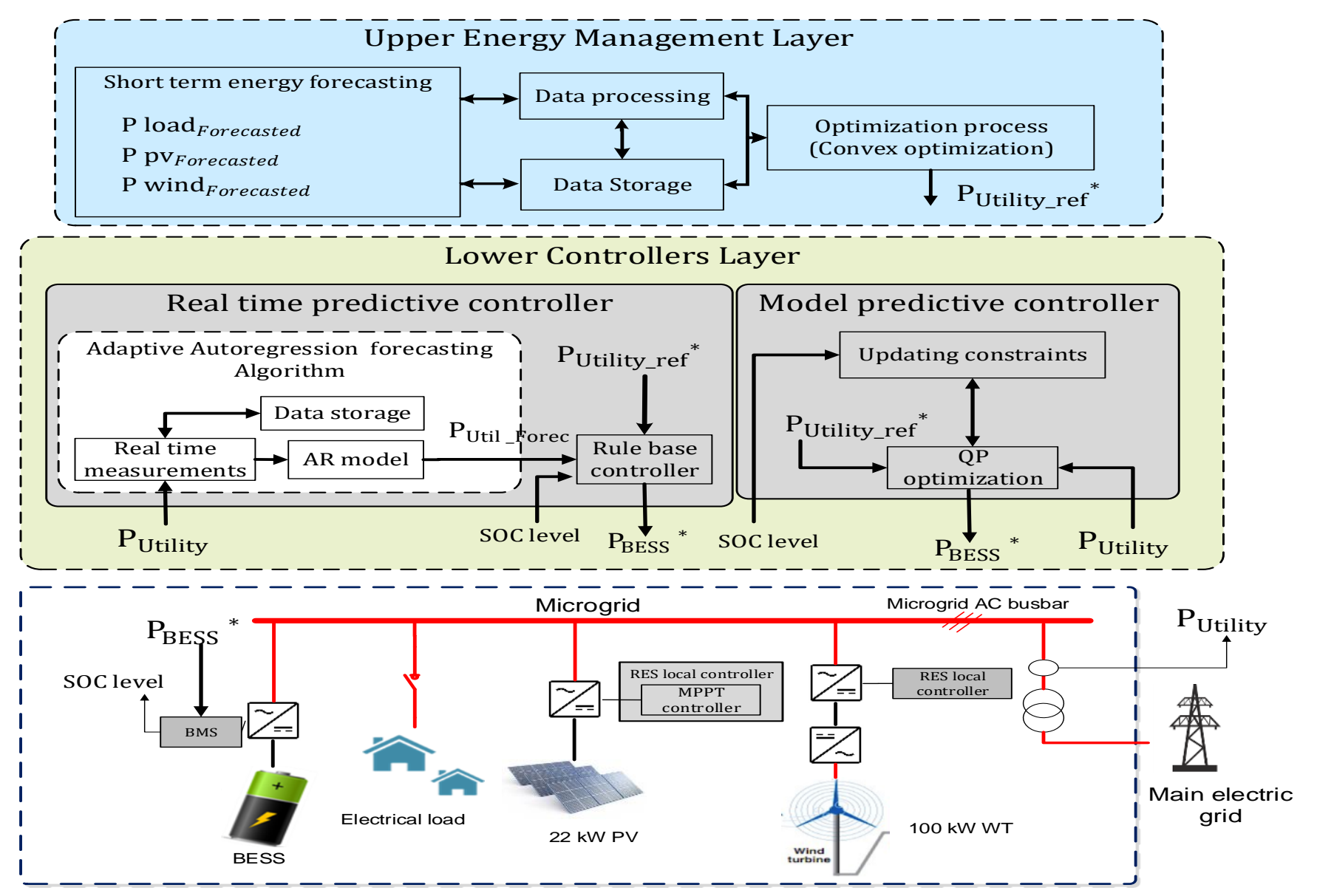

Fig. 1. The overall control hierarchy for the proposed MG

- Constraint for the rate of change of BESS power

This constraint reflects the max ramp up/down rate for the BESS power output between two consecutive time slots.

$$
\Delta \mathrm{P}_{\text {BESS }}(\mathrm{t}) \leq \Delta \mathrm{P}_{\text {BESS } \max }
$$

This constraint is added to smooth the control of the BESS and avoid sharp changes in BESS power to keep the life time of BESS as long as possible.

\subsection{Convex Programming}

Convex optimization is a subfield of optimization that studies the problem of minimizing convex functions over convex sets [38]. Convex minimization has a lot of applications in a wide range of disciplines, such as automatic control systems, data analysis and modeling, and structural optimization [39]. The Convex optimization technique is a recently introduced approach to optimization not previously applied to MGEM. It achieves more accurate results and also uses less computing time compared to linear programming or MILP programming techniques which are typically used to solve MG cost optimization problems [40]. The role of the optimization is to find the best solution for the objective function in the set of solutions that satisfy the constraints (constraints can be equations, inequalities or linear restrictions on the type of a variable) [41], [42]. In this research, convex optimization has been used to solve the linear optimization problem formulated. Since linear functions are convex, linear programming problems are also convex problems. The mathematical formulation of the convex problem is expressed as follows:

$$
\begin{array}{cl}
\text { minimize } & f_{0}(x) \\
\text { subject to } & g_{i}(x) \leq \mathrm{b}_{\mathrm{i}}, \quad \mathrm{i}=1, \ldots, \mathrm{m} \\
& h_{i}(\mathrm{x})=0, \quad \mathrm{i}=1, \ldots, \mathrm{p}
\end{array}
$$

where $x \in R^{n}$, the functions $f_{0}(x)$ and $g_{i}(x)$ must be convex, and the function $f_{m}(x)$ must be "affine" [38]

In this research, the Interior-point method is used since in practice it has been shown to achieve good results [43]. The main advantage of using the convex optimization technique is the processing time: it needs only 5 minutes to perform the optimization process and determine the best reference values Also, convex optimization can easily be programmed because it is very similar to linear programming.

\section{Lower Control Layer}

The main functions of this layer are: (1) force the MG to accurately follow the reference values obtained from the UEML, (2) smooth the power profile for the power drawn by the MG from the main grid, (3) control the BESS with high accuracy to compensate for any change in load and generation through the day. This is achieved using a RHPC or a MPC that controls the settings of the BESS in real-time using a one minute sample time 


\subsection{Adaptive Autoregression Forecasting Algorithm}

Forecasting of the load demand and RES generation for the next samples time is called very short-term energy forecasting (VSTEF). An Autoregression (AR) algorithm is one of the most popular algorithms in VSTEF [44]. In this work, an Adaptive AR algorithm is used to forecast the net demand for the next sample time. AR is a simple method that can be used to obtain accurate forecasts for time series problems. Energy forecasting using the AR model is based on using a time series model that depends on stochastic calculations in which the future values are predicted based on the past values. As the model uses data from the same input variable at previous time steps to forecast the next value, it is named autoregression. Adaptive AR forecasting is used in this paper as it is a simple method, has a fast computing time (only $25 \mathrm{~ms}$ to compute the forecasted value for the next minute), is adaptive and can be trained easily for the time series used.

The AR model used in this research is defined by the following equation:

$$
\mathrm{y}_{\mathrm{t}}=\Phi+\psi_{1} \mathrm{y}_{\mathrm{t}-1}+\psi_{2} \mathrm{y}_{\mathrm{t}-2}+\cdots+\psi_{\mathrm{p}} \mathrm{y}_{\mathrm{t}-\mathrm{p}}+A_{\mathrm{t}}
$$

where $y_{t}$ is the forecasted value, $\psi_{1}, \psi_{2}, \ldots \psi_{\mathrm{p}}$ and $\Phi$ are coefficients found by optimizing the model on training data, $\mathrm{y}_{\mathrm{t}-1}, \mathrm{y}_{\mathrm{t}-2}, \ldots . \mathrm{y}_{\mathrm{t}-\mathrm{p}}$ are the past series values (lags), $\mathrm{P}$ is the order of the AR model and $A_{t}$ is white noise, assumed zero in this paper. Fig. 2 shows the operation of the adaptive autoregression forecasting algorithm to forecast the net demand of the MG for the next minute. The forecasted net demand by the AR algorithm is used as an input to the RHPC/MPC to determine the BESS settings.

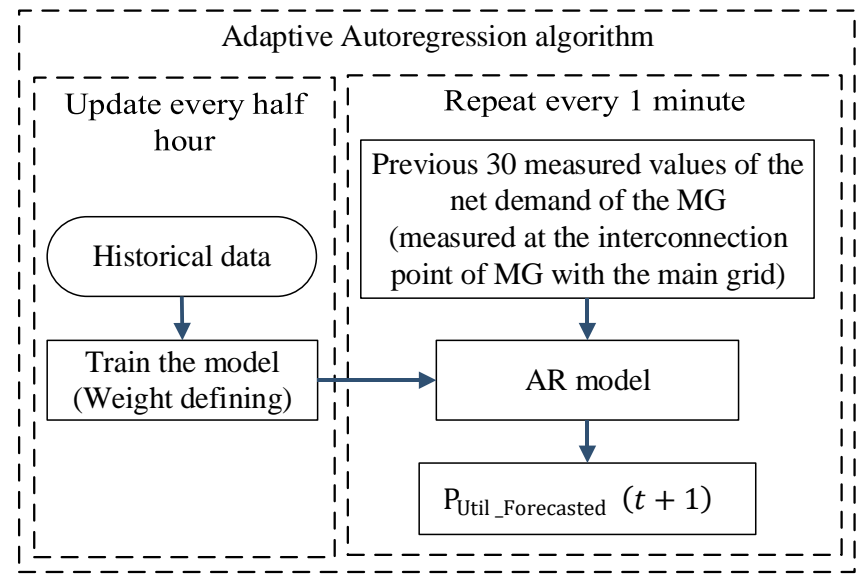

Fig. 2. Adaptive Autoregression forecasting algorithm

\subsection{Rolling Horizon Predictive Controller}

The RHPC depends on a rolling horizon base strategy and a predictive technique. The operating principle of the RHPC is based on determining the correct setting of the BESS in the next time step (i.e. one minute) by predicting accurately the net demand of the MG (Load demand minus PV generation minus wind power generation) in the next time step and comparing it with the reference values for the power drawn from the main electric grid (obtained from the UEML). This process is repeated in a rolling horizon every sample time. Very short-term forecasting is used to complete this operation. The operating procedure of the RHPC is shown in Fig. 3

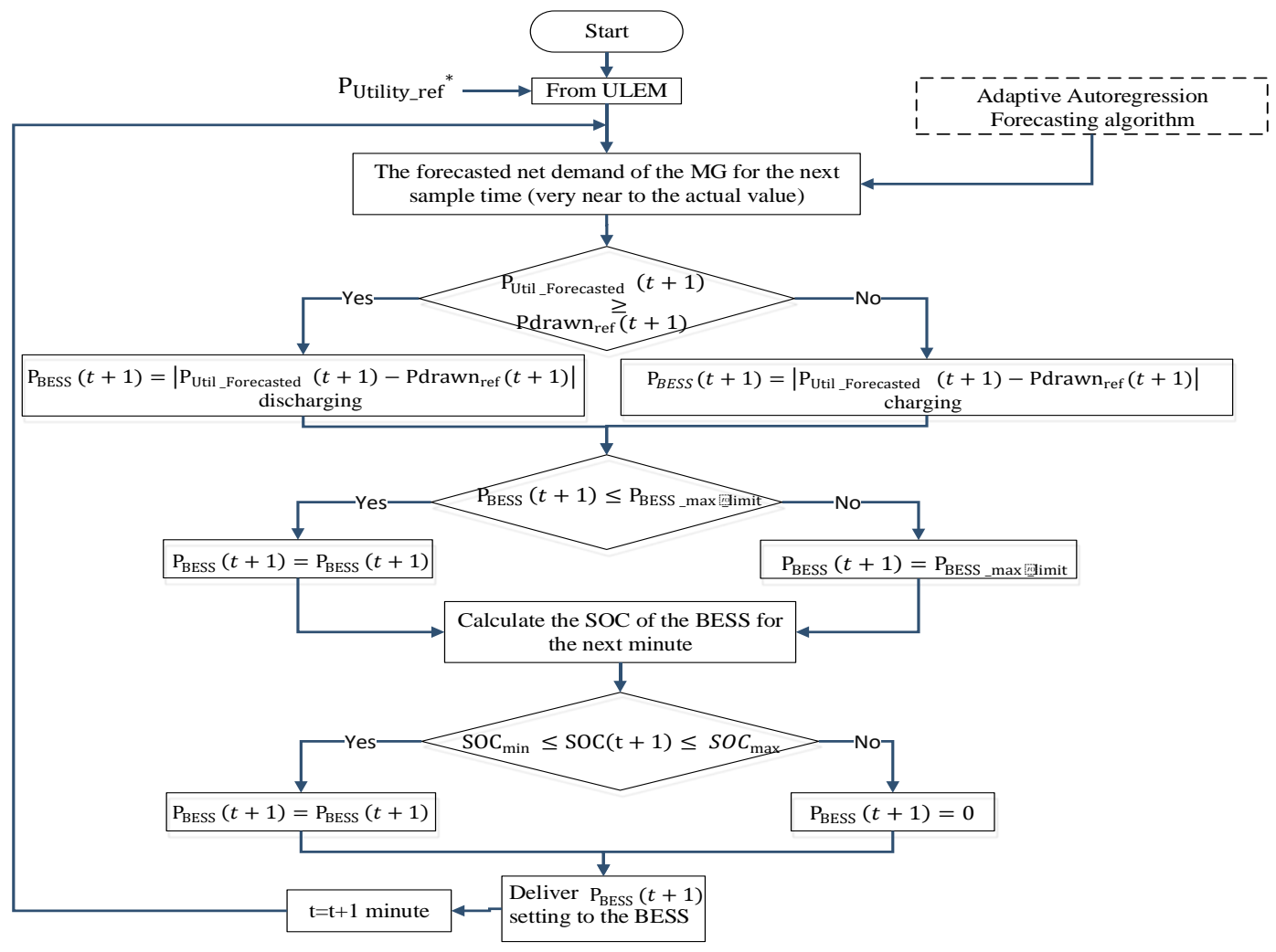

Fig. 3. Operating procedure of the RHPC 


\subsection{Model Predictive Controller}

MPC is an advanced method of process control. MPC has a long history in the field of control engineering where it has been used in chemical plants and oil refineries since 1980 [45],[46]. Recently, it has also been used in power system applications and management [47],[48]. MPC is used to determine the optimal settings for the BESS which force the MG to follow the reference value [49]. The main advantage of MPC is that it optimizes the system for the current sample while keeping account of future changes that will happen. This is achieved by converting the system from a differential equation in continuous to a linear form in discrete time $(13,14)$

$$
\begin{array}{lc}
x_{k+1}=A * x_{k}+B * u_{k} & \mathrm{k}=0,1, \ldots \ldots \mathrm{N}-1 \\
y_{k}=C * x_{k}+D * u_{k} & \mathrm{k}=0,1, \ldots \ldots \mathrm{N}
\end{array}
$$

where $u_{k}$ is the system input at time instant $\mathrm{k}, x_{k+1}, x_{k}$ are the state variables at time instant $\mathrm{k}+1, \mathrm{k}$ respectively, A, B, C, D are the state space model matrices in discrete form, and $\mathrm{y}_{\mathrm{k}}$ is the measured output at time instant $\mathrm{k}$. Equations (5), (7) and (11) have been converted to linear equations. After that, an optimization process over a finite time-horizon is solved.

\subsubsection{MPC Quadratic programming}

This section focuses on the formulation of the MPC control objective (or cost function) which minimizes the difference between the measured output and the reference set point. The Least Squares problem formulation can be used to estimate values of parameters of a mathematical model from measured data, which are subject to errors (15).

$$
\Phi_{z}=\frac{1}{2} \sum_{k=1}^{N}\left\|z_{k}-r_{k}\right\|^{2} Q_{z}
$$

where $\Phi_{z}$ is the control objective (or cost function), $\mathrm{z}_{k}$ is the measured output at time instant $\mathrm{k}, \mathrm{r}_{k}$ is the reference set point at time instant $\mathrm{k}$, and $\mathrm{Q}_{z}$ is the weight matrix

The input constraints are considered limits over the control input signal with maximum and minimum values. Also, a limitation over control signal change between two successive steps should be considered to ensure smooth MPC control.

$$
\begin{gathered}
u_{\min } \leq u \leq u_{\max } \\
\Delta u_{\min } \leq u \leq \Delta u_{\max }
\end{gathered}
$$

To make the least squares form of the problem easier to solve using optimization solvers, it is converted to a Quadratic programming (QP) problem. QP is a special type of nonlinear programming which involves minimizing or maximizing an objective function subject to bounds, linear equality, and inequality constraints. This type of nonlinear programming arises when the objective function is quadratic and the constraints are linear. The problem in QP form can be shown as

$$
\min _{U} \quad \Phi_{z}=\frac{1}{2} U^{T} H_{z} U+g_{z}{ }^{T} U
$$

subjected to the constraints on $U$, where $U$ is the control signal, $U^{T}$ is the vector transpose of $\mathrm{U}, g_{z}$ is a real-valued, ndimensional vector, $H_{z}$ is a $n \times n$ real matrix.

\subsubsection{BESS control using MPC}

The main function of the MPC is to force the MG to follow the reference values for the power drawn from the main grid- obtained from the ULEM. The MPC manipulates the input $\mathrm{P}_{B E S S}(k)$ to achieve an output $\mathrm{P}_{\text {Utility }}(k)$ as close as possible to the setpoint $\mathrm{P}_{\text {Utility_ref }}{ }^{*}$. The measured actual power drawn from the main electric grid $\mathrm{P}_{\text {Utility }}(k)$ is used as a measured output signal (mo), the BESS setting $\left(\mathrm{P}_{B E S S}\right)$ as the input signal (Manipulated variable; $\mathrm{mv}$ ), and $\mathrm{P}_{\text {Utility_ref }}{ }^{*}$ is the reference value (r) for the power drawn from the main electric grid. Constraints over input variables $\left(\mathrm{MV}_{\mathrm{MAX}}, \mathrm{MV}_{\mathrm{MIN}}\right)$ are used to manage the BESS operation. The constraints on the manipulated variable $\left(\mathrm{P}_{B E S S}\right)$ are updated every sample time to keep the BESS operation within its limits. The optimal control signal $\mathrm{P}_{B E S S}(k)$ is determined by an optimization problem at each time instant $(\mathrm{k})$ taking into consideration both the prediction horizon and control horizon parameters. A sample step of 1 minute, a prediction horizon of 15 steps and a control horizon of 15 steps are used. The prediction Horizon $(\mathrm{P})$, is the number of future control periods that the MPC controller must evaluate by prediction when optimizing its control signal at interval $\mathrm{k}$. The control horizon $(\mathrm{C})$, is the number of moves to be optimized at the control interval $\mathrm{k}$. Also, the closed loop performance is adjusted to be compatible with system changes. Fig .4 shows the proposed control approach for the MPC [50]

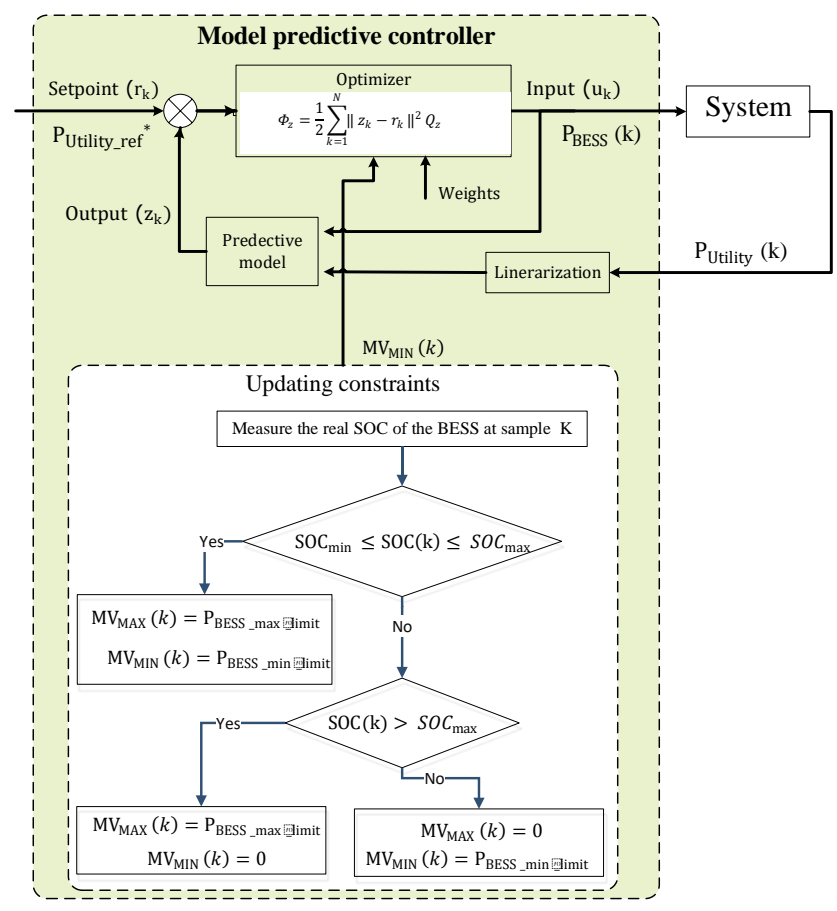

Fig. 4. BESS control algorithm using Model predictive controller 

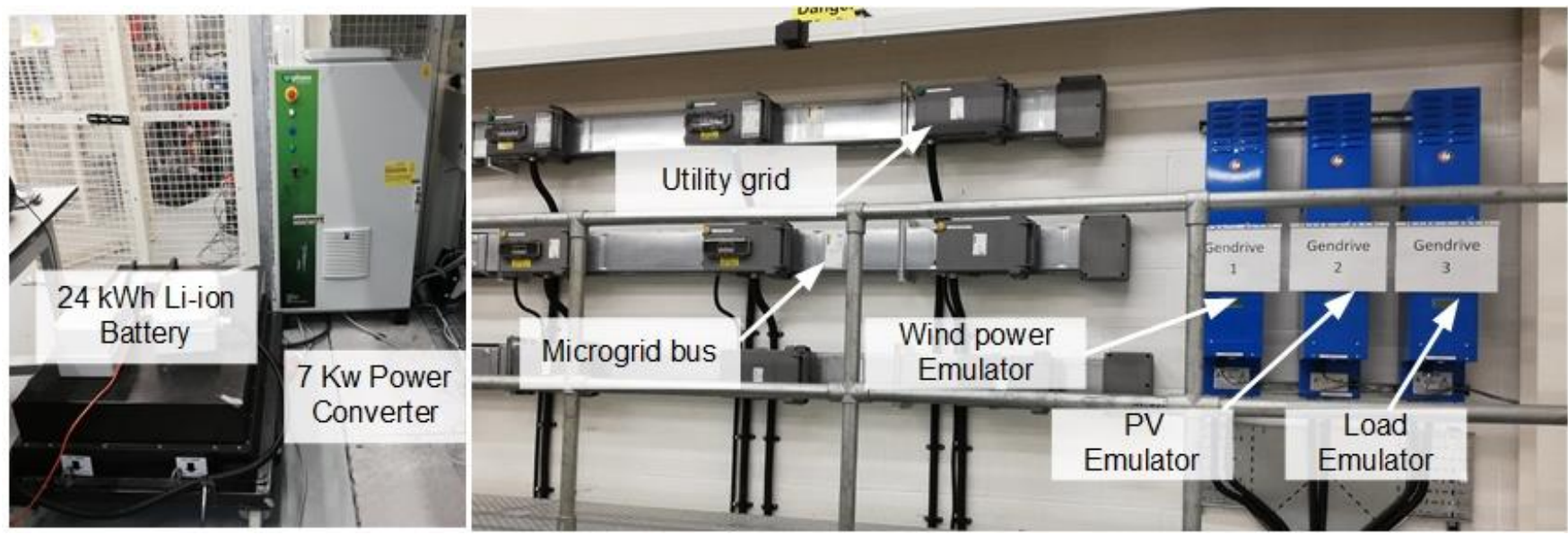

Fig. 5. The complete architecture of the microgrid used in the experiment at the University of Nottingham Laboratory

Table 1

The components of the MG used in this experiment and the used software development packages

\begin{tabular}{|c|c|}
\hline Equipment / Software & Description \\
\hline 2 busbars, $2000 \mathrm{~A}$ each & One busbar is used as the MG busbar, and the other one represents the utility (i.e. main grid). \\
\hline 6 disconnecting switches, $63 \mathrm{~A}$ each & Used to protect the whole MG. \\
\hline Battery energy storage system (BESS) & One BESS consists of a $24 \mathrm{kWh} \mathrm{Li-ion} \mathrm{battery} \mathrm{and} \mathrm{a} 7 \mathrm{~kW}$ power converter to interface with the MG. \\
\hline $\begin{array}{l}\text { CAN bus communication system, } \\
\text { Speed } 1 \mathrm{Mbps}\end{array}$ & $\begin{array}{l}\text { Controller Area Network (CAN) is the communication system used in this experiment, it represents the } \\
\text { nervous system that enables the communication between all MG's parts. }\end{array}$ \\
\hline $1 \mathrm{PC}$ & Core i3-7100 CPU, $3.91 \mathrm{GHz}, 8$ GB RAM \\
\hline Nominal system voltage/ frequency & $380 \mathrm{~V} / 50 \mathrm{HZ}$. \\
\hline LABVIEW software & Used as a graphical user interface GUI and a control tool to implement the proposed control algorithm \\
\hline MATLAB software & Used to run the optimization algorithm that is used in the high-level EM stage. \\
\hline
\end{tabular}

Table 2.

Parameters of the battery energy storage system used

\begin{tabular}{cccc}
\hline Parameter & Rating & Parameter & Rating \\
\hline $\begin{array}{c}\text { Nominal battery } \\
\text { capacity }\end{array}$ & $24 \mathrm{kWh}$ & $\begin{array}{c}\text { Converter rated } \\
\text { power }\end{array}$ & $\pm 7 \mathrm{~kW}$ \\
$\begin{array}{c}\text { Nominal battery } \\
\text { voltage }\end{array}$ & $400 \mathrm{~V}$ & $\begin{array}{c}\text { Converter fixed } \\
\text { losses }\left(\mathrm{P}_{\mathrm{C}_{\text {_conv }}}\right)\end{array}$ & $0.33 \mathrm{~kW}$ \\
$\begin{array}{c}\text { Battery efficiency } \\
\left(\eta_{\mathrm{d}}\right)\end{array}$ & $90 \%$ & $\mathrm{C}_{\mathrm{deg}}$ & $10^{-9} £ / \mathrm{W}$ \\
$\begin{array}{c}\text { Converter } \\
\text { efficiency }\left(\eta_{\text {Conv }}\right)\end{array}$ & $95 \%$ & $\mathrm{~N}_{\text {cycle }}$ & 4000 \\
SOC $_{\min }$ & $20 \%$ & $\mathrm{CC}_{\text {bat }}$ & $380 £ / \mathrm{kWh}$ \\
SOC $_{\max }$ & $90 \%$ & & \\
\hline
\end{tabular}

\section{Energy management results}

The complete EM strategy has been implement in real time including: (1) the UEML, which determines the reference values for the daily power drawn by the MG from the main electric grid, (2) the LCL, in which RHPC or MPC is used to determine the control settings for the BESS in real time using reference values obtained from the UEML, and a periodic measurement of the real SOC of the BESS and the real net demand of the MG. In this experiment, the load profile, PV generation and wind profiles have been scaled down to be suitable for use in the laboratory since the MG available at the laboratory has a limited rating for its components.
Table 3.

Purchasing and selling electricity tariffs

\begin{tabular}{|c|c|c|}
\hline Type & Time & Value \\
\hline $\begin{array}{l}\text { Off-peak purchasing } \\
\text { tariff }\end{array}$ & From 12 am to 7 am & 5 pence $/ \mathrm{kWh}$ \\
\hline $\begin{array}{c}\text { Mid-peak purchasing } \\
\text { tariff }\end{array}$ & From 7 am to $4 \mathrm{pm}$ & 12 pence/kWh \\
\hline Peak purchasing tariff & From $4 \mathrm{pm}$ to $8 \mathrm{pm}$ & 21 pence/kWh \\
\hline $\begin{array}{l}\text { Mid-peak purchasing } \\
\text { tariff }\end{array}$ & From $8 \mathrm{pm}$ to $12 \mathrm{am}$ & 12 pence/kWh \\
\hline Fixed selling tariff & All day & 4.85 pence/kWh \\
\hline
\end{tabular}

5.1. Laboratory-based microgrid architecture and parameters.

The experimental system was implemented in the University of Nottingham FlexElec Laboratory, using the microgrid shown in Fig. 4. The components of the MG used in this experiment and the software development packages used in this research are listed in Table 1. Also, the parameters of the battery energy storage system used are listed in Table 2.

The microgrid is connected to an isolated busbar. The main source for this MG is a $90 \mathrm{kVA}$ Triphase converter [51] which is a programmable source acting as the main grid connection. Three $10 \mathrm{~kW}$ bidirectional Gendrive power converters are connected between the main utility bus and the MG: these inject or absorb power and reactive power into the 
MG according to references received from a CANBUS communication interface [52]. They are used to emulate the load profiles, PV profiles, and wind power profiles by following references (generated using the load, PV, and wind power data described in section 2) sent from the central control platform. A $24 \mathrm{kWh}$ battery system is also connected to the MG using a $7 \mathrm{~kW}$ Triphase power converter. The reference for the battery again is received from the central control platform via a CANBUS interface. The central control platform is the hierarchical control structure presented in this paper, implemented using LABVIEW on a $\mathrm{PC}$ and communicating with all MG elements.

\subsection{Implementation of the MGEM in the Laboratory}

The UEML is implemented using LABVIEW software which includes embedded MATLAB functions to perform the convex optimization process. This optimization determines the best operating scenario for the MG for one day ahead to minimize the daily operating cost of the MG and increase the self-consumption of the RES. The reference settings for the power drawn from the main grid, obtained from the optimization process, are updated and passed to the LCL every 15 minutes using a CAN communication system. The optimization process uses a 15 minutes sample time. And is repeated every 15 minutes in a rolling horizon manner. The tariff schemes used in this optimization process are a TOU tariff for purchasing electric energy from the main grid, and a fixed tariff for selling electric energy to the main grid [53], [54]. The values of these tariffs are shown in Table 3 . The forecasted daily profiles for the load demand and are feed to the optimization process using a sample time of 15 minutes. The forecasted load and generation profiles are obtained from another forecasting package (i.e. Adaptive neuro-fuzzy inference system (ANFIS) has been used as the forecasting tool in the UEML) [55], [56], [57]. All the forecasted profiles are of 15 minutes sampling time. The average MAPE of the daily forecasted profiles for the load demand, PV generation and Wind power generation are $11.2 \%, 19.6 \%$, and $15.4 \%$.

The LCL includes two controllers: (1) RHPC, (2) MPC. Only one controller is used per experiment.

The RHPC/MPC is implemented using LABVIEW software which includes embedded MATLAB functions to execute the rule base controller and $\mathrm{AR}$ forecasting algorithms.. The main function of the RHPC/MPC in this simulation is to make the $\mathrm{MG}$ accurately follow the reference values for the power drawn from the main grid in real time. Every sample time (i.e. one minute), the RHPC/MPC:

- Receives the reference values for the power drawn by the MG from the main electric grid from the UEML.

- Receives the measured SOC of the BESS using the CAN system.

- Receives the measured net demand of the MG and uses the integrated adaptive AR algorithm to forecast the MG's net demand for the next sample times.

- Determines and delivers the accurate settings to the BESS every one minute
Fig. 6 shows the experimental results obtained using the UEML and the LCL. Fig. 6a shows the real load profile, real $\mathrm{PV}$ and wind generation profiles for one day. These profiles have a one minute sample time. These profiles are fed to the MG system through the Gendrive emulators to emulate the real load consumption, $\mathrm{PV}$, and wind generation.

Both Fig. 6(b-1) and Fig. 6(c-1) show the reference values for the power absorbed by the MG from the main grid - obtained from the UEML-and also the measured power absorbed by the MG from the main grid through the day in case of using RHPC and MPC respectively. This power is measured at the point of coupling between the MG and the main grid (i.e. in the experiment, the $90 \mathrm{~kW}$ Triphase converter is used in this process). It is obvious from Fig. 6(b1) and Fig. 6(c-1) that the RHPC/MPC succeeded in forcing the MG to follow the reference values for the power drawn from the main grid. The effect of prediction and model uncertainties appears clearly in Fig. 6(b-1) and Fig. 6(c-1). Using a one minute sampling time for the LCL (RHPC or MPC) decreases the effect of the prediction and model uncertainties which leads to a very small difference - in range of 2-5\% - between the actual power absorbed by the MG from the main and the UEML reference. This value is very small and has a negligible effect on the total operating cost for the whole day.

Both Fig. 6(b-2) and Fig. 6(c-2) show the optimal settings sent to the BESS in case of using RHPC and MPC respectively. These settings are sent to the BESS every one minute. Its obvious from figures that the change between any two consecutive settings to the BESS are kept as small as possible to achieve smooth BESS control (avoid charging and discharging the BESS with large changes in power values) to maintain the BESS lifetime. Both Fig. 6(b-3) and Fig. 6(c3 ) show the measured SOC curve of the BESS during the day when using RHPC and MPC respectively. The SOC level is measured every minute and is fed to the RHPC using the CAN communication system and LabVIEW software.

The BESS will be charged during the off-peak time when the cost of purchasing electricity from the main grid is low, and this energy is then used to feed the load during the peak tariff periods. The available PV and wind generation are used to feed part of the loads. If the PV and wind generation exceeds the load demand, the excess energy is stored in the BESS to be used later during peak times. It is obvious from Fig. 6(b-1) and Fig. 6(c-1) that the MG absorbs energy from the main grid during the off-peak time (between 12:00 and 7:00) when the purchase tariff of the electric energy from the main grid is low, and stores this energy in the BESS to be used later during the peak time periods. Fig. 6(b-3) and Fig. 6(c-3) show that the BESS is charged up to $60 \%$ of its capacity during the off-peak tariff period (between 12:00 to 7:00) when the purchase tariff of the electrical energy from the main grid is low, and completed its charging up to $90 \%$ of its capacity using the surplus (free) RES generation (between 7:00 and 16:00) after satisfying the loads. Also, it is observed that the proposed strategy kept the SOC of the BESS within limits (between 20 and $90 \%$ ). 

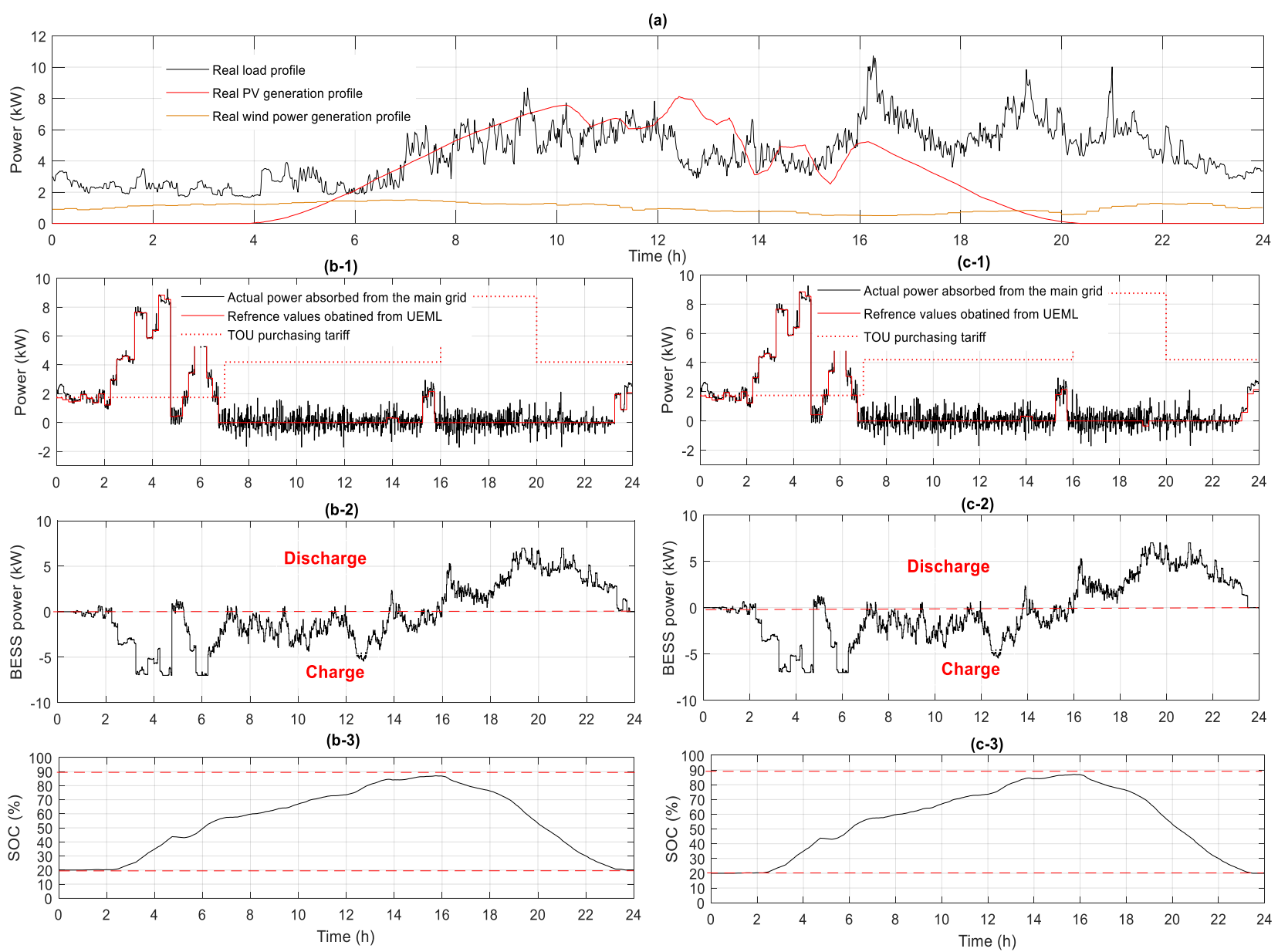

Fig. 6. (a) The real load profile, real PV and wind generation profiles for one day, (b-1) The reference values for the power absorbed from the main grid - obtained from the UEML- and the actual measured power absorbed from the main grid through the day in case of using RHPC, (c-1) The reference values for the power absorbed from the main grid - obtained from the UEML- and the actual measured power absorbed from the main grid in case of using MPC, (b-2), (c-2) are the optimal settings sent to the BESS in case of using RHPC and MPC respectively, (b-3) and (c-3) are the measured SOC curve of the BESS during the day in case of using RHPC and MPC respectively.

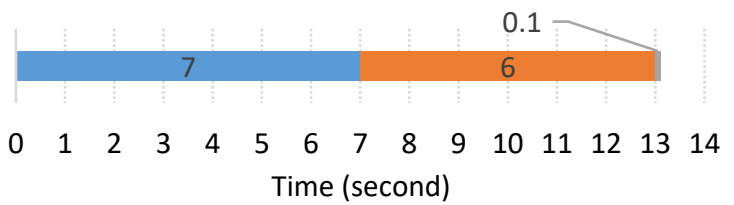

Day ahead forecasting for load demand, PV generation, and wind genration using ANFIS

Convex optimization

Processing data to $\mathrm{LCL}$

(a)

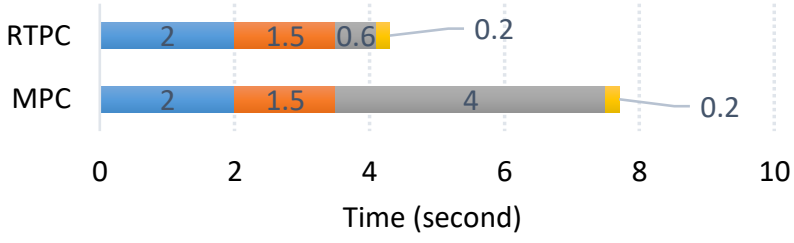

Parallel measurement of load demand, PV
generation, and BESS SOC Autoregression forecasting

Controller computing time

Send optimal setting to BESS

(b)

Fig. 7. The net time required for measuring, computing and communicating through (a) the UEML, (b) the LCL which includes RTPC and MPC.

As the main objective of the EMS is to minimize the daily operating cost of a MG and maximize the self-consumption of the RES within the MG, the EMS has not charged the BESS to its full capacity $(90 \%)$ during the off-peak tariff period, because there is surplus RES generation that could be used to charge the BESS instead of purchasing electric energy from the main grid to charge the BESS even at low tariff. This operating scenario is common when surplus RES (PV generation or wind), is expected. On days where no surplus energy from RES generation is expected, the BESS is charged up to $90 \%$ of its full capacity during the off-peak tariff period (between 12:00 and 7:00) 
Comparing Fig. 6(b-1) and Fig. 6(c-1), there almost no difference between using the RHPC and MPC in the LCL. The only difference is the time used for computing and processing. The MPC requires more time to perform the optimization process to get the best settings of the BESS taking into account the expected variation in the system for the next 15 samples. However, the RTPC uses a rule base controller which takes into account the variation of the next sample point only.

Fig. 6 shows the net time required for measuring, computing and communicating through both the UEML and the LCL which includes RTPC and MPC. It is obvious from Fig. 6a that the net time required for measuring, computing and communicating through UEML is 13.1 seconds only. This time is very short and does not affect the EMS results comparing to the time period in which this layer is updated (i.e. updated every 15 minutes). Fig. $6 \mathrm{~b}$ shows the net time required for measuring, computing and communicating through the LCL. The RTPC uses 4.3 seconds only compared to the MPC which uses 7.7 seconds. As this layer is repeated every 1 minute, the time consumed for measuring, computing and communicating is acceptable and does not affect the accuracy of the results.

From the results obtained it is recommended to use a simple and fast controller (i.e. RTPC) for this LCL since it provides fast response, and has a limited computational time. Using the MPC which performs the optimization process takes more time without achieving more enhanced results.Table 4 shows a comparison between the yearly operating cost of the MG grid, as well as the yearly percentage of self-consumption of the RES when using the proposed EMS compared to other cases. It is obvious from the table that using the two-layer EMS achieves better results compared to the other cases. The results obtained encourage investment in the EMS/BESS as it ensures a reduction in the total operating cost of the MG.

Table 4. A comparison between the yearly operating costs of the MG, as well as the yearly RES self-consumption percent using the proposed EMS and other cases

\begin{tabular}{|l|c|c|c|c|}
\hline \multicolumn{1}{|c|}{ Case } & \multicolumn{2}{|c|}{ Without using rolling horizon } & \multicolumn{2}{c|}{ Using Rolling horizon } \\
\hline & $\begin{array}{c}\text { Yearly operation cost } \\
\text { of the MG (f) }\end{array}$ & $\begin{array}{c}\text { Yearly RES self - } \\
\text { consumption (\%) }\end{array}$ & $\begin{array}{c}\text { Yearly operation } \\
\text { cost of the MG (£) }\end{array}$ & $\begin{array}{c}\text { Yearly RES self - } \\
\text { consumption (\%) }\end{array}$ \\
\hline Without EMS or storage system. & 3941.4 & $51.32 \%$ & 3941.4 & $51.32 \%$ \\
\hline Single layer EMS of 1 hour sample time. & 2751.3 & $67.5 \%$ & 2736.3 & $81.7 \%$ \\
\hline Single layer EMS of half an hour sample time. & 2710.7 & $72.4 \%$ & 2698.7 & $82.5 \%$ \\
\hline Single layer EMS of 15 minutes sample time. & 2688.2 & $79.7 \%$ & 2673.2 & $88.65 \%$ \\
\hline $\begin{array}{l}\text { Two-layer EMS; UEML of 15 minutes sample } \\
\text { time, and LCL of 1 minute sample time * }\end{array}$ & 2671.7 & $83.8 \%$ & 2648.8 & $91.25 \%$ \\
\hline $\begin{array}{l}\text { Ideal case (perfect forecasting and ideal EMS } \\
\text { system). }\end{array}$ & 2562.6 & $95.67 \%$ & 2562.6 & $95.67 \%$ \\
\hline
\end{tabular}

* There are additional income from smoothing the shared power profile with the main grid should be deducted from the total yearly operating costs

\section{Conclusion and future work.}

This paper has presented a new EMS which minimizes the total daily operating cost of microgrids. A complete model for the MG has been built, considering all the constraints that affect the operation of the MG. The convex optimization technique successfully obtains reference values with a low processing time. The results show that the proposed EMS, with two-layer structure, succeeded in reducing the total daily operating cost of the MG and increasing the self-consumption of the RES. A daily operating cost reduction of $18 \%$ to $30 \%$ can be achieved depending on the tariff scheme used, load and generation profiles and on the BESS capacity.

The use of the RHPC and MPC enables the MG to accurately follow the reference values for the power absorbed from the main electric grid. A simple comparison was made to show the merits of two different low layer controllers RHPC and MPC - with the RHPC being judged to be better as it does not require long computational time.

Although using a sample time of one minute is considered a great challenge since it needs a very fast processing time from the controllers, it helps in achieving very accurate result in helping the MG follows the reference values. The experimental results demonstrate the proposed strategy can work in real time with high accuracy.

Future work; the proposed EMS will be tested using a real-time pricing scheme. The effect of incorporating the cost curves of the storage/generation system will be examined. More studies will be included regarding the communication and measurement systems and their effect on the results, especially when partial or complete communication failure occurs for a short period of time. Also, the effect of using demand side management techniques will be studied.

\section{Acknowledgment}

This work was supported by the Egyptian Governmentministry of higher education (cultural affairs and missions sector) and the British Council through Newton-Mosharafa fund. The work has also received support from the University of Nottingham through its Hermes fund.

Also, I would like to thank Richard Davies, Research Associate at the University of Nottingham for his assistance with the experimental work.

\section{References}

[1] Xie M, Ji X, Hu X, Cheng P, Du Y, Liu M. Autonomous optimized economic dispatch of active distribution system with multi-microgrids. Energy. 2018;153:479-89. 
[2] Nejabatkhah F, Li YW. Overview of power management strategies of hybrid AC/DC microgrid. IEEE Transactions on Power Electronics. 2015;30:7072-89.

[3] Roldán-Blay C, Escrivá-Escrivá G, Roldán-Porta C, Álvarez-Bel C. An optimisation algorithm for distributed energy resources management in micro-scale energy hubs. Energy. 2017;132:126-35. [4] Aboli R, Ramezani M, Falaghi H. Joint optimization of dayahead and uncertain near real-time operation of microgrids. International Journal of Electrical Power \& Energy Systems. 2019;107:34-46.

[5] Ebrahimi MR, Amjady N. Adaptive robust optimization framework for day-ahead microgrid scheduling. International Journal of Electrical Power \& Energy Systems. 2019;107:213-23.

[6] Olivares DE, Mehrizi-Sani A, Etemadi AH, Cañizares CA, Iravani $\mathrm{R}$, Kazerani $\mathrm{M}$, et al. Trends in microgrid control. IEEE Transactions on smart grid. 2014;5:1905-19.

[7] Hu M, Xiao J-W, Cui S-C, Wang Y-W. Distributed real-time demand response for energy management scheduling in smart grid. International Journal of Electrical Power \& Energy Systems. 2018;99:233-45

[8] Arcos-Aviles D, Pascual J, Marroyo L, Sanchis P, Guinjoan F. Fuzzy logic-based energy management system design for residential grid-connected microgrids. IEEE Transactions on Smart Grid. 2018;9:530-43.

[9] Pfeifer A, Dobravec V, Pavlinek L, Krajačić G, Duić N. Integration of renewable energy and demand response technologies in interconnected energy systems. Energy. 2018;161:447-55.

[10] Bahramirad S, Reder W, Khodaei A. Reliability-constrained optimal sizing of energy storage system in a microgrid. perspectives. 2012;1:3.

[11] Moradi H, Esfahanian M, Abtahi A, Zilouchian A. Optimization and energy management of a standalone hybrid microgrid in the presence of battery storage system. Energy. 2018;147:226-38.

[12] Bedi AS, Ahmad MW, Swapnil S, Rajawat K, Anand S. Online algorithms for storage utilization under real-time pricing in smart grid. International Journal of Electrical Power \& Energy Systems. 2018;101:50-9.

[13] Koutsopoulos I, Tassiulas L. Control and optimization meet the smart power grid: Scheduling of power demands for optimal energy management. Proceedings of the 2nd International Conference on Energy-efficient Computing and Networking: ACM; 2011.p. 41-50. [14] Farsangi AS, Hadayeghparast S, Mehdinejad M, Shayanfar H. A novel stochastic energy management of a microgrid with various types of distributed energy resources in presence of demand response programs. Energy. 2018;160:257-74.

[15] Wang Y, Mao S, Nelms RM. Distributed online algorithm for optimal real-time energy distribution in the smart grid. IEEE Internet of Things Journal. 2014;1:70-80.

[16] Silvente J, Kopanos GM, Pistikopoulos EN, Espuña A. A rolling horizon optimization framework for the simultaneous energy supply and demand planning in microgrids. Applied Energy. 2015;155:485-501.

[17] Kou P, Liang D, Wang J, Gao L. Stable and Optimal Load Sharing of Multiple PMSGs in an Islanded DC Microgrid. IEEE Transactions on Energy Conversion. 2018;33:260-71.

[18] Kou P, Liang D, Gao L. Distributed coordination of multiple PMSGs in an islanded DC microgrid for load sharing. IEEE Transactions on Energy Conversion. 2017;32:471-85.

[19] Palma-Behnke R, Benavides C, Lanas F, Severino B, Reyes L, Llanos J, et al. A microgrid energy management system based on the rolling horizon strategy. IEEE Transactions on smart grid. 2013;4:996-1006.

[20] Parisio A, Rikos E, Glielmo L. A model predictive control approach to microgrid operation optimization. IEEE Transactions on Control Systems Technology. 2014;22:1813-27.

[21] Marietta MP, Graells M, Guerrero JM. A rolling horizon rescheduling strategy for flexible energy in a microgrid. Energy
Conference (ENERGYCON), 2014 IEEE International: IEEE. p. 1297-303.

[22] Borghetti A, Bosetti M, Bossi C, Massucco S, Micolano E, Morini A, et al. An energy resource scheduler implemented in the automatic management system of a microgrid test facility. 2007 International Conference on Clean Electrical Power: IEEE; 2007. p. 94-100.

[23] Korpas M, Holen AT. Operation planning of hydrogen storage connected to wind power operating in a power market. IEEE Transactions on Energy Conversion. 2006;21:742-9.

[24] Luna AC, Meng L, Diaz NL, Graells M, Vasquez JC, Guerrero JM. Online energy management systems for microgrids: experimental validation and assessment framework. IEEE Transactions on Power Electronics. 2018;33:2201-15.

[25] Logenthiran T, Srinivasan D. Short term generation scheduling of a microgrid. TENCON 2009-2009 IEEE Region 10 Conference: IEEE; 2009. p. 1-6.

[26] Xiaoping L, Ming D, Jianghong H, Pingping H, Yali P. Dynamic economic dispatch for microgrids including battery energy storage. The 2nd International Symposium on Power Electronics for Distributed Generation Systems: IEEE; 2010. p. 914-7.

[27] Arcos-Aviles D, Pascual J, Guinjoan F, Marroyo L, Sanchis P, Marietta MP. Low complexity energy management strategy for grid profile smoothing of a residential grid-connected microgrid using generation and demand forecasting. Applied energy. 2017;205:6984.

[28] Pascual J, Sanchis P, Marroyo L. Implementation and control of a residential electrothermal microgrid based on renewable energies, a hybrid storage system and demand side management. Energies. 2014;7:210-37.

[29] de Matos JG, e Silva FS, Ribeiro LAdS. Power control in ac isolated microgrids with renewable energy sources and energy storage systems. IEEE Transactions on Industrial Electronics. 2015;62:3490-8.

[30] Richardson I, Thomson M. Domestic electricity demand model-simulation example. Data Sets and Software (CREST). 2010. [31] ETB_UoN_Notts_UK. Daily ETB_UoN_Notts_UK 22kW. Available online : https://pvoutput.org.

[32] company E. WIND-POWER GENERATION DATA. Avaialable online: https://www.elia.be/en/grid-data/powergeneration/wind-power 2018.

[33] Elkazaz MH, Hoballah AA, Azmy AM. Operation optimization of distributed generation using artificial intelligent techniques. Ain Shams Engineering Journal. 2016;7:855-66.

[34] Arora P, White RE, Doyle M. Capacity fade mechanisms and side reactions in lithium - ion batteries. Journal of the Electrochemical Society. 1998;145:3647-67.

[35] Garcia-Torres F, Bordons C. Optimal economical schedule of hydrogen-based microgrids with hybrid storage using model predictive control. IEEE Transactions on Industrial Electronics. 2015;62:5195-207.

[36] Li Z, Huang J, Liaw BY, Zhang J. On state-of-charge determination for lithium-ion batteries. Journal of Power Sources. 2017;348:281-301

[37] Hu X, Li SE, Yang Y. Advanced machine learning approach for lithium-ion battery state estimation in electric vehicles. IEEE Transactions on Transportation electrification. 2015;2:140-9.

[38] Ben-Tal A, Nemirovski A. Robust convex optimization. Mathematics of operations research. 1998;23:769-805.

[39] Kim S-J, Giannakis GB. An online convex optimization approach to real-time energy pricing for demand response. IEEE Transactions on Smart Grid. 2017;8:2784-93.

[40] Severini M, Pisacane O, Fagiani M, Squartini S. Collaborative Energy Management in Micro-Grid environments through multiobjective optimization. 2018 International Joint Conference on Neural Networks (IJCNN): IEEE; 2018. p. 1-8.

[41] Jünger $M$, Liebling TM, Naddef D, Nemhauser GL, Pulleyblank WR, Reinelt $\mathrm{G}$, et al. " 50 Years of Integer 
Programming 1958-2008: From the Early Years to the State-of-theart": Springer Science \& Business Media; 2009.

[42] Elkazaz MH, Hoballah A, Azmy AM. Optimizing distributed generation operation for residential application based on automated systems. Electric Power and Energy Conversion Systems (EPECS), 2015 4th International Conference on: IEEE; 2015. p. 1-6.

[43] Boyd S, Vandenberghe L. Convex optimization: Cambridge university press; 2004.

[44] Baharudin Z, Kamel N. Autoregressive method in short term load forecast. Power and Energy Conference, 2008 PECon 2008 IEEE 2nd International: IEEE; 2008. p. 1603-8.

[45] Holkar K, Waghmare L. An overview of model predictive control. International Journal of Control and Automation. 2010;3:47-63.

[46] Wang L. A Tutorial on Model Predictive Control: Using a Linear Velocity - Form Model. Asia - Pacific Journal of Chemical Engineering. 2004;12:573-614.

[47] Xing X, Xie L, Meng H. Cooperative energy management optimization based on distributed MPC in grid-connected microgrids community. International Journal of Electrical Power \& Energy Systems. 2019;107:186-99.

[48] Martin JA, Hiskens IA. Corrective Model-Predictive Control in Large Electric Power Systems. IEEE Transactions on Power Systems. 2017;32:1651-62.

[49] Wang Y, Boyd S. Fast model predictive control using online optimization. IEEE Transactions on control systems technology. 2010;18:267-78.
[50] Alejandro J, Arce A, Bordons C. Combined environmental and economic dispatch of smart grids using distributed model predictive control. International Journal of Electrical Power \& Energy Systems. 2014;54:65-76.

[51] Triphase converter data is available online https://triphase.com/microgrid-converters/.

[52] Li F, Wang L, Liao C. CAN (Controller Area Network) bus communication system based on Matlab/Simulink. Wireless Communications, Networking and Mobile Computing, 2008 WiCOM'08 4th International Conference on: IEEE; 2008. p. 1-4. [53] purchasing electricity tariffs in UK Available online: https://www.greenenergyuk.com

[54] Selling electricity tariffs in UK. Available at: https://www.gov.uk/feed-in-tariffs.

[55] Laouafi A, Mordjaoui M, Dib D. One-hour ahead electric load forecasting using neuro-fuzzy system in a parallel approach. Computational Intelligence Applications in Modeling and Control: Springer; 2015. p. 95-121.

[56] Elena Dragomir O, Dragomir F, Stefan V, Minca E. Adaptive Neuro-Fuzzy Inference Systems as a strategy for predicting and controling the energy produced from renewable sources. Energies. 2015;8:13047-61.

[57] Kassa Y, Zhang J, Zheng D, Wei D. Short term wind power prediction using ANFIS. 2016 IEEE International Conference on Power and Renewable Energy (ICPRE): IEEE; 2016. p. 388-93. 